\title{
Rapid and label-free detection of COVID-19 using coherent anti-Stokes Raman scattering microscopy
}

\author{
Tanveer A. Tabish, UCL Cancer Institute, University College London, London, Bloomsbury WC1E 6DD, UK \\ Roger J. Narayan, Joint Department of Biomedical Engineering, University of North Carolina and North Carolina State University, Raleigh, \\ NC 27599-7115, USA \\ Mohan Edirisinghe, Department of Mechanical Engineering, University College London, Torrington Place, London WC1E 7JE, UK \\ Address all correspondence to Roger J. Narayan at roger_narayan@unc.edu
}

(Received 18 August 2020; accepted 26 October 2020)

\begin{abstract}
From the 1918 influenza pandemic (H1N1) until the recent 2019 severe acute respiratory syndrome coronavirus 2 (SARS-CoV-2) pandemic, no efficient diagnostic tools have been developed for sensitive identification of viral pathogens. Rigorous, early, and accurate detection of viral pathogens is not only linked to preventing transmission but also to timely treatment and monitoring of drug resistance. Reverse transcriptionpolymerase chain reaction (RT-PCR), the gold standard method for microbiology and virology testing, suffers from both false-negative and false-positive results arising from the detection limit, contamination of samples/templates, exponential DNA amplification, and variation of viral ribonucleic acid sequences within a single individual during the course of the infection. Rapid, sensitive, and label-free detection of SARS-CoV2 can provide a first line of defense against the current pandemic. A promising technique is non-linear coherent anti-Stokes Raman scattering (CARS) microscopy, which has the ability to capture rich spatiotemporal structural and functional information at a high acquisition speed in a label-free manner from a biological system. Raman scattering is a process in which the distinctive spectral signatures associated with lightsample interaction provide information on the chemical composition of the sample. In this prospective, we briefly discuss the development and future prospects of CARS for real-time multiplexed label-free detection of SARS-CoV-2 pathogens.
\end{abstract}

\section{Introduction}

Coronaviruses (CoVs) are lipid-enveloped, single-stranded, positive-sense ribonucleic acid (RNA) viruses that belong to the coronaviridae family. ${ }^{[1]} \mathrm{CoVs}$ are roughly pleomorphic or spherical (80-220 nm in size) with obvious spike glycoproteins, which form a crown-like appearance under electron microscopy. ${ }^{[2]}$ CoVs induce a wide spectrum of animal and human diseases. The CoV subfamily contains four genera, namely alpha-CoVs, beta-CoVs, gamma-CoVs, and delta-CoVs. Phylogenetically, severe acute respiratory syndrome coronavirus (SARS-CoV-2) belongs to the betacoronavirus genus. ${ }^{[2]}$ The mortality rate of SAR-CoV-2 is higher than other respiratory viral infections such as Middle East respiratory syndrome-CoV (MERS-CoV). ${ }^{[3]}$ The spike glycoprotein of SARS-CoV-2 binds to the angiotensinconverting enzyme 2 (ACE2) receptor as a cell entry pathway; SARS-CoV-2 uniquely replicates the RNA genome via the production of a nested set of viral RNA pathogens. Replication of the viral RNA genome in airway epithelial cells triggers cell damage and chronic inflammation. ${ }^{[4]}$ Like SARS-CoV, SARS-CoV-2 remains stable, specifically on plastic or stainless steel surfaces (up to days) and in aerosols (up to $3 \mathrm{~h}$ ). ${ }^{[5]}$ Airborne droplets and direct contact are thought to be major routes of SARS-CoV-2 transmission among humans. The distinct medical signs of SARS-CoV-2 include nasal congestion, persistent cough, fever with chills, breathing difficulties, and pneumonia. ${ }^{[5]}$ The virus structure, metabolism, replication, maturation processes, and interaction with the host system have been schematically illustrated by Astuti et al. ${ }^{[3]}$ The pathology of SARS-CoV-2 largely remains unknown; however, new data on this topic are anticipated based on ongoing intensive scientific research. Based on the novelty of the viral genome and the rapid viral replication rate, the existence of accurate and efficient diagnostic methods remains an unanswered question.

The currently available diagnostic modality for the detection of SARS-CoV-2 is reverse transcription-polymerase chain reaction (RT-PCR). ${ }^{[6]}$ In real-time RT-PCR, amplification of target RNA is monitored with the progression of the PCR reaction. Reverse transcription is an RNA-dependent DNA polymerase, which transforms the viral genomic RNA template into complementary single-stranded DNA (cDNA). cDNA, which is more stable than RNA, serves as a template to transform the single-stranded cDNA into the double-stranded DNA. There are two kinds of cDNA synthesis primers, including non-specific sequences (hexamer or oligo-dT) and specific sequences (DNA labeled with both fluorescence and quenching agents). ${ }^{[7]}$ The objective of RT-PCR is to amplify a cDNA sequence based on the RNA template. The sensitivity of the PCR process is dependent upon the primer and probe designs, 
RNA extraction, as well as optimization of the PCR assay. A wide range of RT-PCR assays were designed during the SARS-CoV epidemic, such as fluorescent dyes and sequence-specific primers (e.g., DNA labeled with fluorescent dyes), which generate fluorescence or electrical signals with minimal contamination of sample/template. ${ }^{[8]}$

RT-PCR testing is particularly vulnerable to false-negative and false-positive results. False-negative results are generally associated with the specimen source, degradation of the nucleic acid, the detection limit, mutations in viral RNA genome, amplification inhibitors, missing fusion variants, and an inappropriate amount of organisms in the sample. ${ }^{[7,9]}$ False-positive results arise from DNA contamination in the genomic RNA, binding of primer to contaminated DNA, and non-sequencing at low temperature. ${ }^{[10]}$ In China, false-negative results have been reported at rates as high as $20 \%$ to $40 \%$, which raised serious concern over the use of RT-PCR. ${ }^{[11]}$ To improve the efficacy of RT-PCR testing, a number of parameters should be taken into account, such as primer design, halflife, stability, and immunogenicity of the RNA genome, the distance between the primers and DNA polymerase, the relationship between disease severity and analyte concentration, and monitoring of mutations (and specimens) over several time points (from both the upper and lower respiratory tracts). PCR testing is generally very expensive and time consuming. RNA extraction from swab samples takes more than $4 \mathrm{~h}$; more than $24 \mathrm{~h}$ is needed to accomplish the lengthy process of PCR testing and analysis. The advantages and limitations of other diagnostic methods for COVID-19 loop-mediated isothermal amplification (LAMP assay) and protein testing have been reviewed elsewhere. ${ }^{[12]}$ Table I summarizes currently available techniques with sample source, assay time, sensitivity, operating temperature, and limitations for the detection of SARS-COV-2.

A cost-effective, point-of-care, rapid, sensitive, and multiplexed approach for label-free detection of SARS-CoV-2 in patients remains unavailable. Importantly, a combination of diagnostic tools and clinical validation can facilitate outbreak management and reduce inaccurate results. A promising solution is Raman spectroscopy. Spontaneous Raman spectroscopy (also known as linear Raman spectroscopy) is a versatile analytical method that provides spectral information about the molecular vibrational states of the chemical structure of a molecule via photon scattering. ${ }^{[21]}$ Vibrational spectroscopy (both Raman and infrared) has extensively been used as a point-of-care biomedical tool for the diagnosis of human diseases, such as cancer, ${ }^{[2]}$ cardiovascular disease, ${ }^{[23]}$ bacterial infection, ${ }^{[24]}$ fungal infection, ${ }^{[25]}$ and viral ${ }^{[26]}$ infections. However, the fingerprints of chemical structures of biological molecules obtained using spontaneous Raman scattering are very weak in comparison to the incident photon. Therefore, non-linear coherent anti-Stokes Raman scattering (CARS) was introduced to address these limitations of weak signals by manipulating enormous electromagnetic field enhancement resulting from the four-wave mixing phenomena of non-linear optics. ${ }^{[27]}$ CARS has been widely exploited for biological analysis (e.g., tumor detection and biomedical imaging). We have recently reported on the use of CARS to study the antimicrobial activity of graphene-composites ${ }^{[28]}$; our data provide compelling evidence regarding the use of this technique for the detection of viral strains. Raman spectroscopy coupled with non-linear optics has the potential to detect SARS-CoV-2; it

Table I. Comparison of molecular and immunological diagnostic methods to detect SARS-CoV-2.

\begin{tabular}{|c|c|c|c|c|c|c|}
\hline Technique & Sample source & Assay time & Sensitivity & Temperature & Limitations & Ref. \\
\hline RT-PCR & Swab & $2-4 \mathrm{~h}$ & $95 \%$ & $\begin{array}{l}\text { Thermal } \\
\text { cycling }\end{array}$ & Expensive and time-consuming & [13-16] \\
\hline $\begin{array}{l}\text { Loop-mediated } \\
\text { isothermal } \\
\text { amplification } \\
\text { (LAMP) }\end{array}$ & $\begin{array}{l}\text { Throat swabs in the case } \\
\text { of LAMP; while } \\
\text { nasopharyngeal } \\
\text { aspirates in the case of } \\
\text { RT-LAMP }\end{array}$ & $15-60 \mathrm{~min}$ & $99 \%$ & $60-65^{\circ} \mathrm{C}$ & $\begin{array}{l}\text { Time-consuming, expensive, } \\
\text { difficulty associated with the } \\
\text { design of appropriate primers, } \\
\text { false-positive results, and low } \\
\text { throughput }\end{array}$ & {$[14,17]$} \\
\hline $\begin{array}{l}\text { Immunoassay } \\
\text { methods to detect } \\
\text { viral antigens } \\
\text { (e.g., ELISA) }\end{array}$ & Serum & $3-5 h$ & $99 \%$ & Ambient & $\begin{array}{l}\text { Time-consuming, limitation of } \\
\text { detection of antigen, } \\
\text { vulnerable to contamination } \\
\text { and false-positive results }\end{array}$ & [18] \\
\hline $\begin{array}{l}\text { Immunoassay } \\
\text { methods to detect } \\
\text { antibodies } \\
\text { (e.g., ELISA) }\end{array}$ & Serum & $3-5 h$ & $67-98 \%$ & Ambient & $\begin{array}{l}\text { Expensive and antibody } \\
\text { instability }\end{array}$ & {$[14,19]$} \\
\hline $\begin{array}{l}\text { Next-generation } \\
\text { sequencing }\end{array}$ & $\begin{array}{l}\text { Blood, respiratory } \\
\text { samples, and fluid }\end{array}$ & - & - & Ambient & $\begin{array}{l}\text { Time-consuming, expensive, } \\
\text { and requirement for technical } \\
\text { expertise }\end{array}$ & {$[15,20]$} \\
\hline
\end{tabular}


can pave the way for rapid label-free diagnosis and continuous monitoring of SARS-CoV-2 disease.

\section{Non-linear Raman scattering for the detection of coronavirus infections}

Vibrational modes of a molecule encode a wealth of structural and conformational information. Inelastic scattering of photons from matter, known as the Raman effect, is used to directly measure the molecular vibrations of a molecule with visible or near-infrared (NIR) light. ${ }^{[21]}$ Raman spectroscopy is a powerful technique to identify the unique vibrational states of a biological system by measuring the change in frequency of the incident light. Most Raman scattering is measured by the Stokes shift. In spontaneous Raman scattering, 1 in $10^{8}$ incident photons undergoes the Raman effect. ${ }^{[29]}$ Therefore, it is known as incoherent Raman scattering. Furthermore, Raman imaging using the spontaneous Raman effect requires high power laser sources to image a small cross-section; as such, several hours are required to image a cell. ${ }^{[27]}$ Spontaneous Raman scattering also becomes irrelevant for the measurement of molecules at very low concentrations. The weak light-matter interaction and the long exposure time limit its application in clinical settings. The polarization phenomena in spontaneous Raman scattering obeys first-order (linear) optics, which is a two-wave mixing process. ${ }^{[21]}$ These challenges in spontaneous Raman microscopy have been overcome by multiphoton microscopy using an approach that is known as CARS.

CARS has revolutionized the field of vibrational spectroscopy by probing vibrational coherences in biological systems in a label-free manner. CARS is a non-linear four-wave mixing process. CARS uses two synchronized pulsed light sources, a pump field, and a probe field of frequencies, $\omega_{\mathrm{p}}$ and $\omega_{\mathrm{pr}}$, respectively, which are mixed with a third field of Stokes frequency, $\omega_{\mathrm{s}}$, to coherently excite the molecules and create an anti-Stokes field of frequency $\omega_{\mathrm{as}}=\omega_{\mathrm{p}}-\omega_{\mathrm{s}}+\omega_{\mathrm{pr}}{ }^{\left[{ }^{[30]}\right.}$ The frequency of one laser is fixed; the frequency of the other is tunable such that the beat frequency $\left(\omega_{\mathrm{p}}-\omega_{\mathrm{s}}\right)$ coincides with the frequency of Raman-active vibrational modes of the sample. The use of coherent laser fields in CARS generates strong signals and enables a three-dimensional view of molecular vibrations and native fingerprints in unstained samples. ${ }^{[31]}$ The energy diagrams of spontaneous Raman spectroscopy and CARS are shown in Fig. 1. Chemically specific imaging of biological systems using CARS circumvents the problems of photobleaching (associated with fluorescence imaging), chemical perturbation (by exogenous fluorescent labels), and long excitation wavelength (in infrared imaging). ${ }^{[32]}$ The spatiotemporal overlapping of pump and Stokes fields are strictly centered on the unstained molecule, resulting in a signal with a minimal excitation volume at incredibly rapid scanning rates. CARS microscopy has several advantages over traditional imaging modalities, such as high penetration depth into molecules (up to several hundred micrometers in intact samples), minimal photodamage to samples, unstained sampling, high spatiotemporal resolution, as well as label-free and chemically specific multiplexing.

In 1999, Zumbusch et al. developed three-dimensional CARS with a colinear pump, Stokes pump, and near-infrared ultrashort laser system for the imaging of live cells. ${ }^{[33]}$ Over the last 20 years, CARS has extensively been exploited for biomedical applications, such as the detection of lipid-based prostate circulating tumor cells, ${ }^{[34]}$ tumor identification, ${ }^{[35]}$ retinal changes $^{[36]}$ atherosclerosis, ${ }^{[37]}$ the presence of pharmaceutical agents, ${ }^{[38]}$ and bacterial infections. ${ }^{[39]}$ Heuke et al. ${ }^{[40]}$ reported on the detection and diagnosis of ex vivo non-melanoma skin cancer (NMSC), specifically differentiation of healthy skin from NMSC cells using non-linear optical microscopies such as CARS microscopy as well as second harmonic generation and two-photon excited fluorescence imaging of human cancer samples. Figure 2 [40] illustrates the morphological features of NMSC detection using CARS microscopy and other imaging modalities. Figure 2 shows that the features associated with
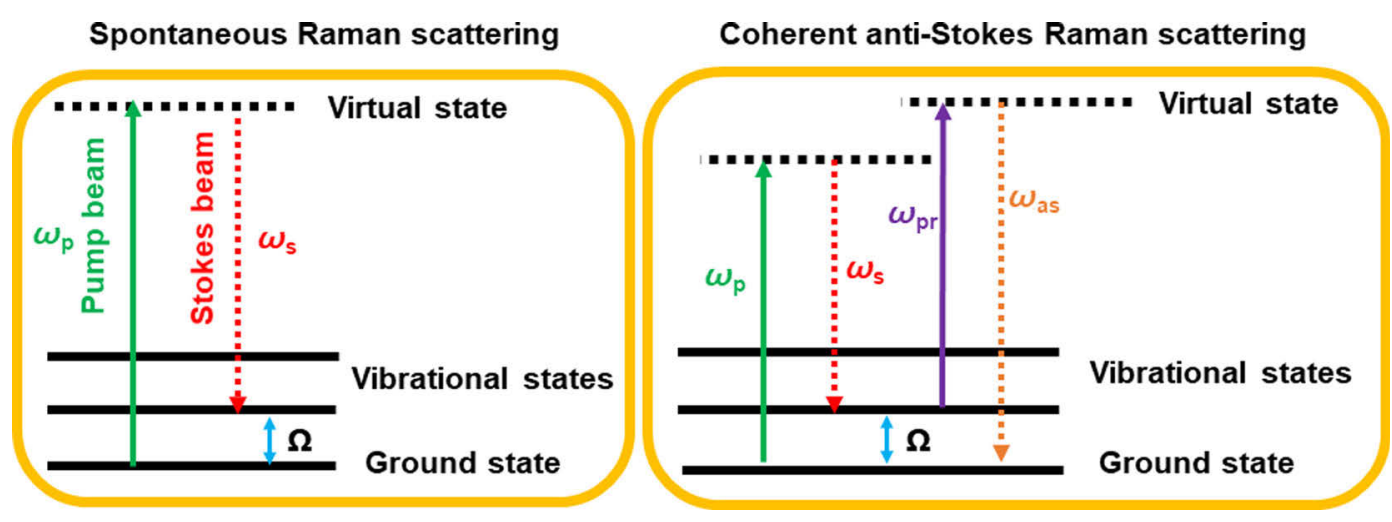

Figure 1. An illustration showing the energy levels of spontaneous and coherent anti-Stokes Raman scattering (CARS). In spontaneous Raman scattering, incoherent emission is triggered by the interaction of the pump with the molecule while in CARS, and coherent emission is mediated by the interaction between the pump and Stokes beams with the molecule. A four-wave mixing non-linear process of CARS uses two pulsed lasers (pump and probe) of frequencies $\omega_{\mathrm{p}}$ and $\omega_{\mathrm{pr}}$ mixed with a Stokes frequency $\omega_{\mathrm{s}}$ to coherently excite the molecule and create an anti-Stokes field of frequency $\omega_{\mathrm{as}}=\omega_{\mathrm{p}}-\omega_{\mathrm{s}}+\omega_{\mathrm{pr}}$. 

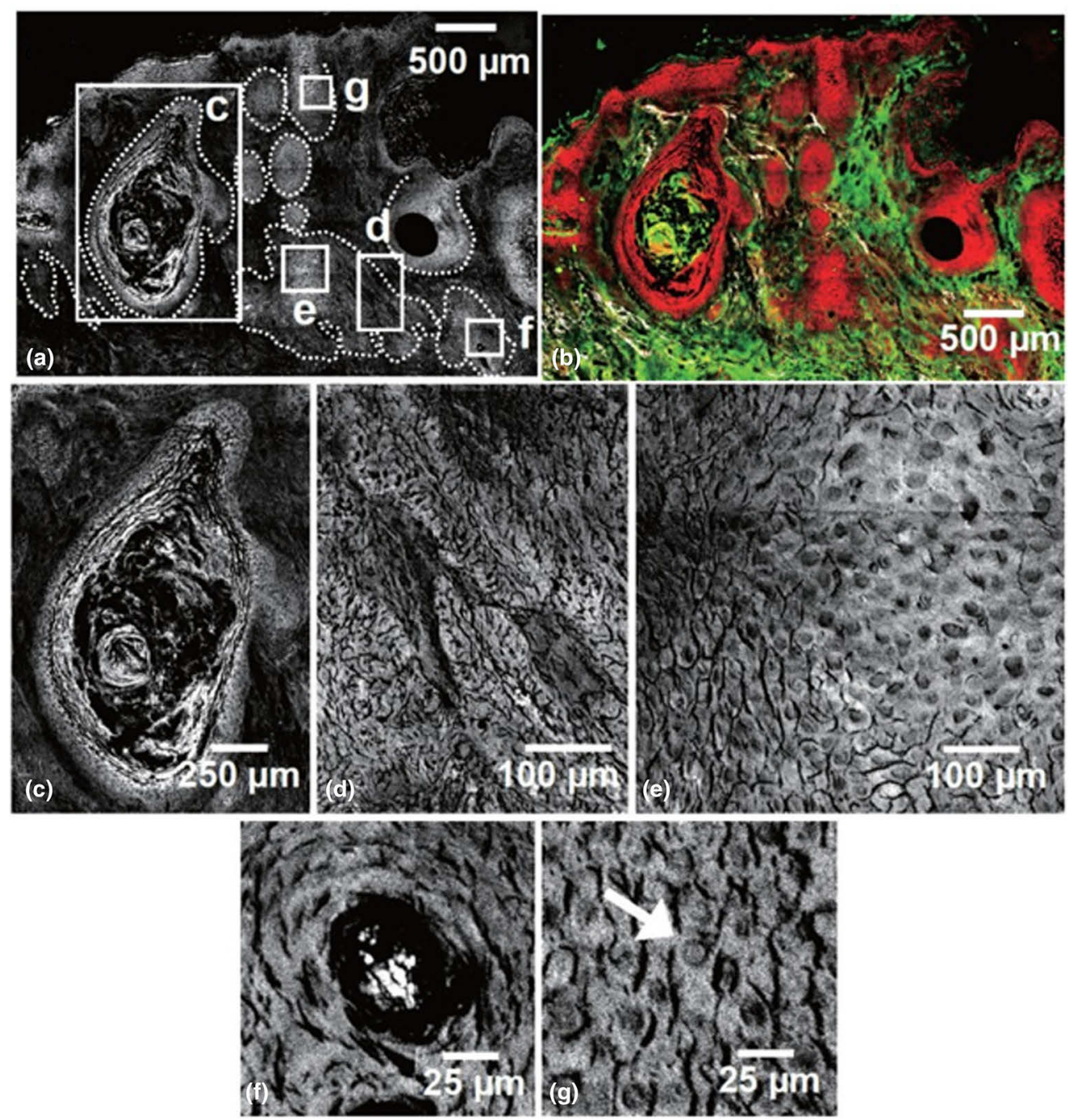

Figure 2. (a) Coherent anti-Stokes Raman scattering (CARS) microscopy image of tumor tissue. (b) CARS image representing the tumor-containing region, which is located by the dotted lines in (a). (c) Keratinizing tumor. (d) Squamous cell carcinoma (SCC) tumor nest. The tumor cells can be discerned from the embedding dermal tissue. (e) Tumor cells with pleomorphic nuclei. (f) Keratin pearl. (g) SCC cells. The white arrow points towards a nucleus, which appeared dark in CARS. Compared with noncancerous tissue, the tumorous tissue possesses an increased cell density, larger nuclei, and an elevated nuclear cytoplasm ratio. Reproduced with permission from Ref. [40] — https://creativecommons.org/licenses/by/3.0/.

the clinical diagnosis of NMSC can efficiently be detected using CARS. CARS has also been used in virology. ${ }^{[41]}$ In 2006, Nan et al. ${ }^{[42]}$ used CARS to detect changes in cellular lipids associated with hepatitis $\mathrm{C}$ virus (HCV). They also combined two-photon fluorescence with CARS to study the subcellular internalization of HCV RNA (using non-diseased cells for RNA replication) and variations in lipid metabolism by HuH-7 hepatoma cells. Lyn et al. ${ }^{[43]}$ investigated the intra- and sub-cellular internalization of HCV and the phenotype of genomic RNA in live cells after antiviral treatment using CARS. They demonstrated the efficiency of CARS to assess the impact of lovastatin on lipid metabolism and also to monitor HCV RNA replicons upon treatment. CARS microscopy is extremely sensitive to the dispersion of viral RNA genomes from primary sites as well as to chemical/structural modifications in lipid droplet size/volume. In another study, Lyn et al. ${ }^{[44]}$ reported on the ability of CARS to quantify overexpression of HCV proteins and reveal alterations in lipid droplet size, density, localization, and redistribution of these proteins. CARS has been explored to detect abnormal lipid metabolism, cholesterol catabolism, and triglyceride excretion events linked to HCV RNA replication ${ }^{[45]}$; changes in lipid phenotype ${ }^{[46]}$; quantitative monitoring of phenotypic variations associated with lipid metabolism and storage in Caenorhabditis elegans (as a model organism for host-virus interactions) ${ }^{[47,48] \text {; }}$ and imaging fibroblast cells infected by cytomegalovirus. ${ }^{[49]}$ Recently, Scully et al. ${ }^{[50]}$ used femtosecond adaptive spectroscopic methods coupled with CARS to illustrate and 
distinguish between influenza and picornavirus with enhanced sensitivity. This work also lends further credence to the diagnostic potential of CARS for viral research.

Lipid droplets have been used as a biomarker for viral infections. CARS has effectively been used as a sensitive tool to study host-virus interactions, cellular membrane membranes, and lipid metabolism mechanisms; these studies have improved our understanding of metabolic disorders in viral infections. The significant role of lipid droplets in the viral lifecycle has long been appreciated. Lipids are the building blocks of viruses that play crucial biological functions in viral lifecycle activities, such as signaling, energy storage, viral replication, endocytosis, and exocytosis. ${ }^{[51]}$ Importantly, it has now been recognized that viruses target lipid signaling and lipid metabolism; viruses fuse with lipid membranes during their entry into host cells, modifying the host cells to support viral envelopment, replication, and maturation. ${ }^{[41]}$ Lipid metabolism is a critical target of SARS-CoV-2 in addition to the ACE2 entry pathway. ${ }^{[51]}$ Several studies have revealed that lipid metabolism enzymes, such as cytosolic phospholipase A2 $\alpha$ enzyme $^{[52]}$ and phospholipase A2 group IID $^{[53]}$, are responsible for the viral RNA genome replication associated with SARS-CoV. The critical role of lipid-mediated pathways in host cells has also been reported for MERS ${ }^{[54,55]}$ and SARS-CoV. ${ }^{[56]}$

The crucial role of lipids in metabolism, replication, and maturation of SARS-CoV-2 can be harnessed as a marker for the early detection of SARS-CoV-2. Although this approach has not been explored to this point, we suggest that CARS can potentially be used as a non-invasive diagnostic method to detect dynamic interactions between various lipid metabolism pathways and RNA replication. Label-free CARS can be used to study lipid metabolism, the levels of expression in infected cells and tissues, as well as the relationships among lipid storage, peroxidation, and desaturation. CARS can provide key insights into the impact of SARS-CoV-2 on the host cell and metabolic events in which lipids within the host cells are modified as a consequence of infection. It has also been proposed that lipid metabolism inhibitors (e.g., statins) can potentially be used to treat SARS-CoV-2. ${ }^{[51]}$ The treatment of COVID-19 is beyond the scope of this Prospective; treatment strategies using a wide range of approaches, such as nanostructures and lipid inhibitors, have been discussed elsewhere. ${ }^{[51,57,58]}$

CARS microscopy has the potential to detect SARS-CoV-2 in a label-free manner. Despite this advantage, CARS microscopy has also some limitations. For example, lipid droplets associated with COVID-positive patients may be affected by underlying conditions. Hence, the characteristic peaks of lipid droplets related to COVID-19 need to be optimized under biologically relevant conditions. Signal optimization process can be carried out by comparing CARS results with standard COVID-19 testing regimens, such as PCR, isothermal amplification, and sequencing. Changes in CARS data associated with the progression of COVID-19 disease need to be understood; it remains unclear whether the coherent Raman-active fingerprints change with the degree of infection. These limitations need to be overcome by performing CARS and surface-enhanced CARS microscopy with clinical samples. By overcoming these limitations, CARS microscopy may represent an innovative label-free molecular diagnostic method for detecting COVID-19 in a timely fashion, which has the potential to complement currently available imaging options in clinical settings.

\section{Summary and future outlook}

SARS-CoV-2 is a more severe human viral pathogen than other viral strains, such as MERS and SARS-CoV-1. The interplay between the lipid-enveloped viral genome and host cells can facilitate the design of innovative strategies to detect, inhibit, and monitor treatment. CARS may enable complex chemical changes that occur between the viral pathogen and the human host to be evaluated. CARS microscopy allows for the unique identification of excitation frequencies and highly sensitive three-dimensional spatiotemporal vibrational imaging of biomolecules. Capturing a myriad of spatiotemporal vibrational modes and phenotypic events of SARS-CoV-2 in real time is one promising use of this technology. To exploit this method in COVID-19 detection, appropriate samples, distinctive Raman-active fingerprints, appropriate sensitivity limit values, and correlations between CARS and the progression of the COVID-19 disease need to be determined. Understanding these parameters for the detection of infection can provide mechanistic insights into transmission pathways and replication of the virus, which in turn can facilitate efforts to limit transmission of the disease. CARS is a non-invasive diagnostic and imaging modality that has the potential to allow for SARS-CoV-2 to be detected in a rapid, label-free manner and the efficacy of therapeutic modalities to be evaluated.

\section{References}

1. L. Ferretti, C. Wymant, M. Kendall, L. Zhao, A. Nurtay, L. Abeler-Dörner, and C. Fraser: Quantifying SARS-CoV-2 transmission suggests epidemic control with digital contact tracing. Science 368, 1-7 (2020).

2. C. Wang, P. Horby, F. Hayden, and G. Gao: A novel coronavirus outbreak of global health concern. Lancet 395, 470-473 (2020).

3. I. Astuti: Severe acute respiratory syndrome coronavirus 2 (SARS-CoV-2): an overview of viral structure and host response. Diabetes Metab. Syndr. 14, 407-412 (2020)

4. P. Mehta, D. McAuley, M. Brown, E. Sanchez, R. Tattersall, and J. Manson: Across speciality collaboration. COVID-19: consider cytokine storm syndromes and immunosuppression. Lancet 395, 1033 (2020).

5. N. Van Doremalen, T. Bushmaker, D. Morris, M. Holbrook, A. Gamble, B. Williamson, and J. Lloyd-Smith: Aerosol and surface stability of SARS-CoV-2 as compared with SARS-CoV-1. N. Engl. J. Med. 382, 1564-1567 (2020).

6. W. Wang, Y. Xu, R. Gao, R. Lu, K. Han, G. Wu, and W. Tan: Detection of SARS-CoV-2 in different types of clinical specimens. JAMA 323, 1843-1844 (2020).

7. Y. Li, L. Yao, J. Li, L. Chen, Y. Song, Z. Cai, and C. Yang: Stability issues of RT-PCR testing of SARS-COV-2 for hospitalized patients clinically diagnosed with COVID-19. J. Med. Virol. 92, 903-908 (2020).

8. S. Emery, D. Erdman, M. Bowen, B. Newton, J. Winchell, R. Meyer, and P. Rota: Real-time reverse transcription-polymerase chain reaction assay for SARS-associated coronavirus. Emerg. Infect. Dis. 10, 311 (2004).

9. A. Xiao, Y. Tong, and S. Zhang: False-negative of RT-PCR and prolonged nucleic acid conversion in COVID-19: rather than recurrence. J. Med. Virol. (2020). doi:10.1002/jmv.25855 
10. A. Tahamtan and A. Ardebili: Real-time RT-PCR in COVID-19 detection: issues affecting the results. Expert Rev. Mol. Diagn. 20, 453-454 (2020).

11.C. Alvarez-Moreno and A. Rodríguez-Morales: Testing dilemmas: post negative, positive SARS-CoV-2 RT-PCR-is it a reinfection? Travel Med. Infect. Dis. (2020). doi:10.1016/j.tmaid.2020.101743.

12. B. Udugama, P. Kadhiresan, H. Kozlowski, A. Malekjahani, M. Osborne, V. Li, and W. Chan: Diagnosing COVID-19: the disease and tools for detection. ACS Nano 14, 3822-3835 (2020).

13. V. Corman, O. Landt, M. Kaiser, R. Molenkamp, A. Meijer, D. Chu, T. Bleicker, S. Brünink, J. Schneider, and M. Schmidt: Detection of 2019 novel coronavirus (2019-nCoV) by real-time RT-PCR. Euro. Surveill. 25, 2000045 (2020).

14. T. Kilic, R. Weissleder, and H. Lee: Molecular and immunological diagnostic tests of COVID-19-current status and challenges. iScience 101406, 1-19 (2020).

15. N. Rabiee, M. Bagherzadeh, A. Ghasemi, H. Zare, S. Ahmadi, Y. Fatahi, and R. Varma: Point-of-use rapid detection of sars-cov-2: nanotechnology-enabled solutions for the COVID-19 pandemic. Int. J. Mol. Sci. 21, 5126 (2020).

16. K. To, O. Tsang, C. Yip, K. Chan, T. Wu, J. Chan, W. Leung, T. Chik, C. Choi, and D. Kandamby: Consistent detection of 2019 novel coronavirus in Saliva. Clin. Infect. Dis. 71, 841-843 (2020).

17. Y. Zhang, N. Odiwuor, J. Xiong, L. Sun, R. Nyaruaba, H. Wei, and N. Tanner: Rapid molecular detection of SARS-CoV2 (COVID-19) virus RNA using colorimetric LAMP. Medrxiv. Posted online February 29, 2020. doi:10.1101/2020.02.26.20028373.

18. B. Freeman, S. Lester, L. Mills, M. Rasheed, S. Moye, O. Abiona, G. Hutchinson, M. MoralesBetoulle, I. Krapinunaya, and A. Gibbons: Validation of a SARS-CoV-2 spike protein ELISA for use in contact investigations and serosurveillance. Biorxiv. Posted online April 25, 2020. doi:10.1101/2020.04.24.057323.

19. SinoBiological: Antigen Detection Assay (2020). https://www.sinobiological.com/research/virus/sars-cov-2-antigen-detection-assay.

20. D. Wang, B. Hu, C. Hu, F. Zhu, X. Liu, J. Zhang, B. Wang, H. Xiang, Z. Cheng, and $Y$. Xiong: Clinical characteristics of 138 hospitalized patients with 2019 novel coronavirus-infected pneumonia in Wuhan, China. JAMA 323, 1061-1069 (2020).

21. P. Martinez Pancorbo, K. Thummavichai, L. Clark, T. Tabish, J. Mansfield, B. Gardner, and Y. Zhu: Novel Au-SiO $-\mathrm{WO}_{3}$ core-shell composite nanoparticles for surface-enhanced Raman spectroscopy with potential application in cancer cell imaging. Advan. Funct. Mat. 29, 1903549 (2019).

22. H. Lui, J. Zhao, D. McLean, and H. Zeng: Real-time Raman spectroscopy for in vivo skin cancer diagnosis. Cancer Res. 72, 2491-2500 (2012).

23. V. Rafalsky, A. Zyubin, E. Moiseeva, and I. Samusev: Prospects for Raman spectroscopy in cardiology. Cardiovas. Therap. Preven. 19, 70-77 (2020).

24. D. Willemse-Erix, M. Scholtes-Timmerman, J. Jachtenberg, W. van Leeuwen, D. Horst-Kreft, T. Schut, and K. Maquelin: Optical fingerprinting in bacterial epidemiology: Raman spectroscopy as a real-time typing method. J. Clin. Microbiol. 47, 652-659 (2009).

25.P. Verwer, W. Van Leeuwen, V. Girard, V. Monnin, A. van Belkum, J. Staab, and W. van de Sande: Discrimination of Aspergillus lentulus from Aspergillus fumigatus by Raman spectroscopy and MALDI-TOF MS. Eur. J. Clin. Microbiol. Infect. Dis. 33, 245-251 (2014).

26. M. Saleem, M. Bilal, S. Anwar, A. Rehman, and M. Ahmed: Optical diagnosis of dengue virus infection in human blood serum using Raman spectroscopy. Laser Phys. Lett. 10, 035602 (2013).

27. J. Cheng and X. Xie: Coherent anti-Stokes Raman scattering microscopy: instrumentation, theory, and applications. J. Phys. Chem B. 108, 827-840 (2004).

28. R. Matharu, T. Tabish, T. Trakoolwilaiwan, J. Mansfield, J. Moger, T. Wu, and M. Edirisinghe: Microstructure and antibacterial efficacy of graphene oxide nanocomposite fibres. J. Colloid Interface Sci. 571, 239-252 (2020).

29. T. Tabish, P. Dey, S. Mosca, M. Salimi, F. Palombo, P. Matousek, and N. Stone: Smart gold nanostructures for light mediated cancer theranostics: combining optical diagnostics with photothermal therapy. Adv. Sci., 7, 1903441 (2020).
30. C. Camp and M. Cicerone: Chemically sensitive bioimaging with coherent Raman scattering. Nat. Photonics 9, 295-305 (2015).

31. A. Lombardini, V. Mytskaniuk, S. Sivankutty, E. Andresen, X. Chen, J. Wenger, and H. Rigneault: High-resolution multimodal flexible coherent Raman endoscope. Light Sci. Appl. 7, 1-8 (2018).

32. R. Jones, D. Hooper, L. Zhang, D. Wolverson, and V. Valev: Raman techniques: fundamentals and frontiers. Nanoscale Res. Lett. 14, 1-34 (2019).

33. A. Zumbusch, G. Holtom, and X. Xie: Three-dimensional vibrational imaging by coherent anti-Stokes Raman scattering. Phys. Rev. Lett. 82, 4142 (1999).

34. R. Mitra, O. Chao, Y. Urasaki, O. Goodman, and T. Le: Detection of lipid-rich prostate circulating tumour cells with coherent anti-Stokes Raman scattering microscopy. BMC Cancer 12, 540 (2012).

35. V. Le, S. Yoo, Y. Yoon, T. Wang, B. Kim, S. Lee, and E. Chung: Brain tumor delineation enhanced by moxifloxacin-based two-photon/CARS combined microscopy. Biomed. Opt. Express 8, 2148-2161 (2017).

36. O. Masihzadeh, D. Ammar, M. Kahook, and T. Lei: Coherent anti-Stokes Raman scattering (CARS) microscopy: a novel technique for imaging the retina. Invest. Ophthalmol. Visual Sci. 54, 3094-3101 (2013).

37. A. Ko, L. Mostaço-Guidolin, A. Ridsdale, A. Pegoraro, M. Smith, V. Slepkov, and M. Sowa: Using multimodal femtosecond CARS imaging to determine plaque burden in luminal atherosclerosis. In Multiphoton Microscopy in the Biomedical Sciences XI, Proceedings of SPIE Vol. 7903 (International Society for Optics and Photonics, 2011) p. 790318.

38. W. Schie, C. Krafft, and J. Popp: Applications of coherent Raman scattering microscopies to clinical and biological studies. Analyst 140, 38973909 (2015).

39. K. Bae, W. Zheng, Y. Ma, and Z. Huang: Real-time monitoring of pharmacokinetics of antibiotics in biofilms with Raman-tagged hyperspectral stimulated Raman scattering microscopy. Theranostics 9, 1348 (2019).

40. S. Heuke, N. Vogler, T. Meyer, D. Akimov, F. Kluschke, J. Röwert-Huber, and J. Popp: Detection and discrimination of non-melanoma skin cancer by multimodal imaging. Healthcare 1, 64-83 (2013).

41. N. Heaton and G. Randall: Multifaceted roles for lipids in viral infection. Trends Microbiol. 19, 368-375 (2011).

42. X. Nan, A. Tonary, A. Stolow, X. Xie, and J. Pezacki: Intracellular imaging of HCV RNA and cellular lipids by using simultaneous two-photon fluorescence and coherent anti-Stokes Raman scattering microscopies. ChemBioChem 7, 1895-1897 (2006).

43. R. Lyn, D. Kennedy, S. Sagan, D. Blais, Y. Rouleau, A. Pegoraro, and J. Pezacki: Direct imaging of the disruption of hepatitis $C$ virus replication complexes by inhibitors of lipid metabolism. Virol. 394, 130-142 (2009).

44. R. Lyn, D. Kennedy, A. Stolow, A. Ridsdale, and J. Pezacki: Dynamics of lipid droplets induced by the hepatitis $C$ virus core protein. Biochem. Biophys. Res. Commun. 399, 518-524 (2010).

45. B. Rakic, S. Sagan, M. Noestheden, S. Bélanger, X. Nan, C. Evans, and J. Pezacki: Peroxisome proliferator-activated receptor $\alpha$ antagonism inhibits hepatitis C virus replication. Chem. Biol. 13, 23-30 (2006).

46. D. Kennedy, R. Lyn, and P. Pezacki: Cellular lipid metabolism is influenced by the coordination environment of copper. J. Am. Chem. Soc. 131, 2444-2445 (2009).

47. T. Hellerer, C. Axäng, C. Brackmann, P. Hillertz, M. Pilon, and A. Enejder: Monitoring of lipid storage in Caenorhabditis elegans using coherent anti-Stokes Raman scattering (CARS) microscopy. Proc. Natl. Acad. Sci. USA 104, 14658-14663 (2007).

48. T. Le, H. Duren, M. Slipchenko, C. Hu, and X. Cheng: Label-free quantitative analysis of lipid metabolism in living Caenorhabditis elegans. J. Lipid Res. 51, 672-677 (2010).

49. I. Robinson, M. Ochsenkühn, C. Campbell, G. Giraud, W. Hossack, J. Arlt, and J. Crain: Intracellular imaging of host-pathogen interactions using combined CARS and two-photon fluorescence microscopies. J. Biophotonics 3, 138-146 (2010).

50. V. Deckert, T. Deckert-Gaudig, D. Cialla, J. Popp, R. Zell, A. Sokolov, and M. Scully: Laser spectroscopic technique for direct identification of a single virus I: FASTER CARS. PNAS 202013169, 1-5 (2020).

51. M. Abu-Farha, T. Thanaraj, M. Qaddoumi, A. Hashem, J. Abubaker, and F. Al-Mulla: The role of lipid metabolism in COVID-19 virus infection and as a drug target. Int. J. Mol. Sci. 21, 3544 (2020). 
52.C. Müller, M. Hardt, D. Schwudke, W. Neuman, S. Pleschka, and J. Ziebuhr: Inhibition of cytosolic phospholipase $A 2 \alpha$ impairs an early step of coronavirus replication in cell culture. J. Virol. 92, e01463-17 (2018).

53. R. Vijay, X. Hua, D. Meyerholz, Y. Miki, K. Yamamoto, M. Gelb, and S. Perlman: Critical role of phospholipase A2 group IID in age-related susceptibility to severe acute respiratory syndrome-CoV infection. J. Exp. Med. 212, 1851-1868 (2020).

54. J. Zhang, Y. Lan, and S. Sanyal: Modulation of lipid droplet metabolism a potential target for therapeutic intervention in flaviviridae infections. Front. Microbiol. 8, 2286 (2017).

55. X. Xu, X. Wu, X. Jiang, K. Xu, L. Ying, C. Ma, and J. Sheng: Clinical findings in a group of patients infected with the 2019 novel coronavirus (SARS-Cov-2) outside of Wuhan, China: retrospective case series. Br. Med. J. 368, m606 (2020).

56. Q. Wu, L. Zhou, X. Sun, Z. Yan, C. Hu, J. Wu, and K. Li: Altered lipid metabolism in recovered SARS patients twelve years after infection. Sci. Rep. 7, 1-12 (2017).

57.T.A. Tabish and M.R. Hamblin: Multivalent nanomedicines to treat COVID-19: a slow train coming. Nano Today 35, 100962 (2020).

58. K. Mansouri, M. Rastegari-Pouyani, M. Ghanbri-Movahed, M. Safarzadeh, and S. Kiani Z: Ghanbari-Movahed: Can a metabolism-targeted therapeutic intervention successfully subjugate coronavirus? A scientific rational. Biomed. Pharmacother 21, 110694 (2020). 